\title{
A nonlinear complementarity problem in mathematical programming in Hilbert space
}

\section{Sribatsa Nanda and Sudarsan Nanda}

In this paper we prove the following existence and uniqueness theorem for the nonlinear complementarity problem by using the Banach contraction principle. If $T: K \rightarrow H$ is strongly monotone and lipschitzian with $k^{2}<2 c<k^{2}+1$, then there is a unique $y \in K$, such that $T y \in K^{*}$ and $(T y, y)=0$ where $H$ is a Hilbert space, $K$ is a closed convex cone in $H$, and $K^{*}$ the polar cone.

1. Introduction and statement of the theorem

Let $H$ be a real Hilbert space and let $K$ be a closed convex cone in $H$ with the vertex at 0 . The polar of $K$ is the cone $K^{*}$, defined by $K^{*}=\{y \in H:(x, y) \geq 0$ for every $x \in K\}$.

A mapping $T: H \rightarrow H$ is said to be monotone on $K$ if $(T x-T y, x-y) \geq 0$ for all $x, y \in K$ and strictly monotone if strict inequality holds whenever $x \neq y, T$ is called strongly monotone if there is a constant $c>0$ such that $(T x-T y, x-y) \geq c\|x-y\|^{2}$. $T$ is said to be lipschitzian if there is a constant. $k>0$ such that $\|T x-T y\| \leq k\|x-y\|$ for all $x, y \in H$ whenever $x \neq y$, and a contraction if $0<k<1$.

The purpose of this note is to prove the following existence and uniqueness theorem for the nonlinear complementarity problem.

Received 6 March 1979. 
THEOREM. Let $T: K \rightarrow H$ be strongly monotone and lipschitzian with $k^{2}<2 c<k^{2}+1$. Then there is a unique $y_{0}$ such that

$$
y_{0} \in K, T y_{0} \in K^{*} \text {, and }\left(T y_{0}, y_{0}\right)=0 \text {. }
$$

\section{Proof of the theorem}

Since $K$ is a nonempty closed convex set in $H$, for every $y \in K$ there is a unique $x \in K$ closest to $y-T y$; that is,

$$
\|x-y+T y\| \leq\|z-y+T y\|
$$

for every $z \in K$. Let the correspondence $y \mapsto x$ be denoted by $\theta$. Let $z$ be any element of $K$ and let $0 \leq \lambda \leq 1$. Since $K$ is convex, $(1-\lambda) x+\lambda z \in K$. Define a function $h:[0,1] \rightarrow R^{+}$by the rule

$$
h(\lambda)=\|y-T y-(1-\lambda) x-\lambda z\|^{2} .
$$

Then $h$ is a twice continuously differentiable function of $\lambda$ and

$$
h^{\prime}(\lambda)=2(y-T y-\lambda z-(1-\lambda) x, x-z) .
$$

Since $x$ is the unique element closest to $y-T y$, we must have $h^{\prime}(0) \geq 0$, and therefore

$$
(y-T y-x, x-z) \geq 0
$$

for every $z \in K$. Let $y_{1}$ and $y_{2}$ be two elements of $K$ and $y_{1} \neq y_{2}$. Let $\theta\left(y_{1}\right)=x_{1}$ and $\theta\left(y_{2}\right)=x_{2}$. Putting $y=y_{1}$ and $z=\theta\left(y_{2}\right)$ in

(2.1) we get

$$
\left(y_{1}-T y_{1}-\theta\left(y_{1}\right), \theta\left(y_{1}\right)-\theta\left(y_{2}\right)\right) \geq 0 .
$$

Again, putting $y=y_{2}$ and $z=\theta\left(y_{1}\right)$ in (2.1), we get

$$
\left(y_{2}-T y_{2}-\theta\left(y_{2}\right), \theta\left(y_{2}\right)-\theta\left(y_{1}\right)\right) \geq 0 \text {. }
$$

From (2.2) and (2.3) we have

$$
\left(y_{1}-T y_{1}-\theta\left(y_{1}\right)-y_{2}+T y_{2}+\theta\left(y_{2}\right), \theta\left(y_{1}\right)-\theta\left(y_{2}\right)\right) \geq 0 .
$$

Hence 


$$
\begin{aligned}
\left(y_{1}-T y_{1}-y_{2}+T y_{2}, \theta\left(y_{1}\right)-\theta\left(y_{2}\right)\right) & \geq\left(\theta\left(y_{1}\right)-\theta\left(y_{2}\right), \theta\left(y_{1}\right)-\theta\left(y_{2}\right)\right) \\
& =\left\|\theta\left(y_{1}\right)-\theta\left(y_{2}\right)\right\|^{2} .
\end{aligned}
$$

Therefore,

$$
\begin{aligned}
\left\|\theta\left(y_{1}\right)-\theta\left(y_{2}\right)\right\|^{2} & \leq\left|\left(y_{1}-T y_{1}-y_{2}+T y_{2}, \theta\left(y_{1}\right)-\theta\left(y_{2}\right)\right)\right| \\
& \leq\left\|y_{1}-T y_{1}-y_{2}+T y_{2}\right\|\left\|\theta\left(y_{1}\right)-\theta\left(y_{2}\right)\right\| .
\end{aligned}
$$

Thus

$$
\left\|\theta\left(y_{1}\right)-\theta\left(y_{2}\right)\right\| \leq\left\|T y_{1}-T y_{2}-y_{1}+y_{2}\right\|
$$

Since $T$ is strongly monotone and lipschitzian, it follows from the inequality (2.4) that

$$
\begin{aligned}
\left\|\theta\left(y_{1}\right)-\theta\left(y_{2}\right)\right\|^{2} & \leq\left\|T y_{1}-T y_{2}-y_{1}+y_{2}\right\|^{2} \\
& =\left(T y_{1}-T y_{2}-y_{1}+y_{2}, T y_{1}-T y_{2}-y_{1}+y_{2}\right) \\
& =\left\|T y_{1}-T y_{2}\right\|^{2}+\left\|y_{1}-y_{2}\right\|^{2}-2\left(T y_{1}-T y_{2}, y_{1}-y_{2}\right) \\
& \leq\left(k^{2}+1-2 c\right)\left\|y_{1}-y_{2}\right\|^{2} .
\end{aligned}
$$

Since $k^{2}<2 c<k^{2}+1$, we have $0<k^{2}+1-2 c<1$. Putting $a^{2}=k^{2}+1-2 c$ in the above inequality we obtain

$$
\left\|\theta\left(y_{1}\right)-\theta\left(y_{2}\right)\right\| \leq a\left\|_{1}-y_{2}\right\|
$$

where $0<a<1$. Thus $\theta$ is a contraction. Now applying the Banach contraction principle (see, for example, [1]) we conclude that $\theta$ has a unique fixed point, say $y_{0}$. Now putting $y=y_{0}$ in (2.1) we get

$$
\left(T y_{0}, z-y_{0}\right) \geq 0
$$

for every $z \in K$. Since $0 \in K$ we have from (2.5) that $\left(T_{y_{0}}, y_{0}\right) \leq 0$. Again since $K$ is a convex cone, $2 y_{0} \in K$ and therefore putting $z=2 y_{0}$ in (2.5) we get $\left(T y_{0}, y_{0}\right) \geq 0$. Thus $\left(T y_{0}, y_{0}\right)=0$ and $\left(T y_{0}, z\right) \geq 0$ for every $z \in K$, showing that $T y_{0} \in K^{*}$. Therefore $y_{0}$ is the unique solution to the complementarity problem (1.1) and this completes the proof. 
Reference

[1] Casper Goffman, George Pedrick, First course in functional analysis (Prentice/Hall of India, New Delhi, 1974).

Department of Mathematics,

Regional Engineering College,

Rourkela,

Orissa,

India. 